\title{
EFFECTS OF ACTIVE AND COLD-WATER IMMERSION RECOVERY STRATEGIES ON PERCEIVED WELL-BEING AND PHYSICAL READINESS: A CROSSOVER STUDY CONDUCTED AFTER SMALL-SIDED SOCCER GAMES
}

original paper

() Wroclaw University of Health and Sport Sciences

DOI: https://doi.org/10.5114/hm.2022.107979

\section{MOHAMMAD KARAMI BASERI ${ }^{1 \oplus}$, JALIL REISI $^{1 \oplus}{ }^{\oplus}$, REZA BAGHERI $^{1 \oplus}$, FILIPE MANUEL CLEMENTE ${ }^{2,3}$}

${ }^{1}$ Department of Exercise Physiology, Faculty of Sport Sciences, University of Isfahan, Isfahan, Iran

${ }^{2}$ Escola Superior Desporto e Lazer, Instituto Politécnico de Viana do Castelo, Viana do Castelo, Portugal

${ }^{3}$ Instituto de Telecomunicações, Delegação da Covilhã, Covilhã, Portugal

\section{ABSTRACT}

Purpose. The study investigated the effect of active recovery (AR) and cold-water immersion (CWI) recovery strategies on the speed of recovery after small-sided games (SSGs) in soccer players.

Methods. A crossover design was employed to divide 24 male soccer players from a first division Iranian National League (age: $22.3 \pm 2.6$ years) into 4 experimental conditions: active-active, active-CWI, CWI-active, and CWI-CWI. Heart rate (HR) variations (standard deviation of normal R-R intervals [SDNN], log-transformed root mean square of successive R-R intervals [lnRMSSD]) and self-reported indices (Hooper questionnaire and rate of perceived exertion [RPE]) were measured. Twenty-four hours after SSGs, the players performed one of the recovery strategies. Forty-eight hours after the session, they completed a 20-m sprint test; changes were compared with baseline.

Results. A significant difference in SDNN HR variations between AR and CWI recovery strategies $\left(F=4.86, p=0.03, \eta^{2}=0.31\right)$ was noted. Regarding within-experimental condition changes $\left(F=60.82, p=0.001, \eta^{2}=0.85\right)$, significant differences were detected when comparing data recorded before SSGs and immediately after SSGs $(p=0.001)$, as well as for data recorded before SSGs and immediately after recovery $(p=0.001)$. There was also a significant difference in InRMSSD HR variations when AR and CWI were compared $\left(F=2.41, p=0.033, \eta^{2}=0.29\right)$. Within-experimental condition changes $(F=127.9, p=$ $\left.0.001, \eta^{2}=0.74\right)$ indicated significant differences between data recorded before SSGs and immediately after SSGs $(p=0.001)$, as well as between data recorded before SSGs and immediately after recovery $(p=0.001)$. No significant difference was found between the SDNN index of HR variability for different recoveries $\left(p=0.055, \eta^{2}=0.07\right)$. Moreover, no significant differences were noted between recovery strategies in terms of Hooper index $\left(p=0.832, \eta^{2}=0.11\right)$, RPE $\left(p=0.41, \eta^{2}=0.06\right)$, or 20-m sprint test $\left(p=0.78, \eta^{2}=0.02\right)$.

Conclusions. CWI showed a greater effect than AR in restoring the impaired vagal-related HR variability indices observed immediately after SSGs. However, no advantage was observed between the recovery strategies.

Key words: association football, athletic performance, fatigue, wellness, heart rate variability, sprint

\section{Introduction}

Soccer involves many physical activities, including running at high speeds, changing direction, decreasing or increasing acceleration, jumping, and tackling [1]. Repeatedly performing vigorous activities during soccer matches causes muscular fatigue, which decreases players' performance during the same match or the subsequent one [2,3]. Marked changes in biochemical factors related to exercise-induced muscle damage remain for at least 72 hours after the match. In addition, decreases in physical performance reflect the attenuated neuromuscular function of maximum voluntary contraction, which reduces vertical jump height and sprint speed; these effects can persist for 24-96 hours after the match [3].

Such issues highlight the importance of implementing recovery strategies after soccer matches, which should be carefully designed [4]. Small-sided games (SSGs) are widely used in soccer training sessions

Correspondence address: Jalil Reisi, Department of Exercise Physiology, Faculty of Sport Sciences, University of Isfahan, Azadi Square, Postal code: 8174673441, Isfahan, Iran, e-mail: j.reisi@spr.ui.ac.ir, https://orcid.org/0000-0002-6569-431X

Received: August 25, 2020

Accepted for publication: February 21, 2021

Citation: Baseri MK, Reisi J, Bagheri R, Clemente FM. Effects of active and cold-water immersion recovery strategies on perceived well-being and physical readiness: a crossover study conducted after small-sided soccer games. Hum Mov. 2022; 23(3):120-129; doi: https://doi.org/10.5114/hm.2022.107979. 
because they mimic the dynamic nature of matches, enabling players to improve both technical and tactical skills, while providing significant physiological stimuli to boost performance [5]. SSGs are adjusted versions of a formal game that reduce the number of players, change the pitch dimensions, or modify task constraints in accordance with the main objective of the coach [6]. As intense drill-based exercises, SSGs may have a residual fatigue effect on players, and some concerns have arisen about their impact on athletes' readiness.

Different recovery strategies (e.g., cold-water immersion [CWI], massage, light- to moderate-intensity exercises) have been prescribed to control the impact of training load on players' physical performance and to attenuate its decreases [1]. It has been shown that soccer players use active recovery (AR) and CWI strategies to reach optimal muscle recovery and reduce exercise-induced fatigue [7, 8]. AR might include slow jogging or submaximal training and stretching/mobility exercises [8]. Elias et al. [9] investigated the efficiency of a single exposure to 14 minutes of CWI and contrast water therapy (CWT) on post-exercise recovery in Australian soccer players, revealing that the former intervention reduced muscular pain and fatigue to a greater extent than CWT [9]. In addition, Bastos et al. [10] assessed the potential benefits of CWI and $\mathrm{AR}$ for blood lactate concentrations and heart rate variability (HRV) indices after high-intensity exercise. They reported that AR and CWI improved performance owing to the removal of blood lactate after high-intensity exercise [10].

Despite a lack of data, CWI is known to result in some improvements in post-exercise cardiac autonomic regulation when compared with $\mathrm{AR}$ and passive recovery [10]. Furthermore, AR is not recommended if the aim of the recovery session is to accelerate parasympathetic reactivation [10]. Although most studies on this topic $[10,11]$ have compared the short-term effects of AR and CWI strategies, these strategies are typically used to accelerate muscular recovery, especially if short rest intervals are considered as being applicable only immediately after the match or the day after. For instance, CWI may be used immediately after a match, and AR can be implemented the day after. Coaches might also prefer employing AR immediately after a match, as well as the day after. However, previous studies examined the short-term effects of AR and CWI when performed separately $[9,10]$, while little attention has been paid to the combined use of these strategies.

The number of investigations regarding SSGs has grown in the last decade [5, 12]. The effects of different task conditions (e.g., size of the field, the format of play, number of ball touches, rule changes) on physiological and physical variables have been extensively reported [13]. Most evidence suggests that a greater physiological impact is exerted during small SSGs formats (1 vs. 1 to 3 vs. 3) [13]. The response of heart rate (HR) to training, rate of perceived exertion (RPE), and concentrations of blood lactate are the common variables assessed in physiological analyses [5]. SSGs seem to be advantageous to training routines. However, little is known about the effect of recovery on physiological parameters [12]. Given the popularity of SSGs and the fact that chronic training sessions are frequently scheduled on consecutive days in soccer, much consideration should be given to players' responses to SSGs when designing soccer training procedures [14].

To date, only 1 study [12] has investigated the effects of using different strategies at different intervals (e.g., immediately after, 24 hours after, and 48 hours after SSGs) on the speed of recovery and fatigue reduction. In addition, it has not yet been examined whether the order and combination of these methods accelerate recovery. Incorporating such strategies could help practitioners and coaches to improve the muscular recovery of soccer players. Therefore, the purpose of this study was to compare the effects of 4 different recovery strategies (CWI immediately after SSGs and AR 24 hours after SSGs, CWI immediately after SSGs and CWI 24 hours after SSGs, AR immediately after SSGs and CWI 24 hours after SSGs, and AR immediately after SSGs and AR 24 hours after SSGs) on the speed of recovery in semi-professional soccer players.

\section{Material and methods}

\section{Study design}

The study followed a crossover design. The intervention occurred in the mid-season, precisely 8 weeks after the beginning of the season. In the first phase of the study, all 24 players who were eligible to participate performed the 30-15 Intermittent Fitness Test so that we could measure their aerobic power. Then, each participant was randomly assigned to 1 of 4 crossover experimental conditions based on the combinations of strategies used: active-active (AA), active-CWI (AC), CWI-active (CA), or CWI-CWI (CC). All experimental conditions had training sessions consisting of a warmup followed by a 3 vs. 3 SSG format, with a training regimen of 4 repetitions of 4 minutes, with 3-minute rests [15]. After the session, the players implemented recovery strategies in accordance with their experi- 


\section{HUMAN MOVEMENT}

M.K. Baseri, J. Reisi, R. Bagheri, F.M. Clemente, Active and cold-water immersion recovery strategies

mental conditions. The experiments occurred at the same time to prevent changes in players' circadian rhythms and to ensure similar conditions between moments of assessment. The active strategy consisted of running at $40-50 \%$ of maximal HR (HRmax) for 10 minutes after the first day of SSGs; the CWI strategy consisted of immersion in cold water $\left(10-12^{\circ} \mathrm{C}\right)$ for 10 minutes. Baseline values of HRV, self-reported measurement, RPE, and 20-m sprint tests were recorded. Comparisons between the experimental conditions were tested to identify any potential differences before the intervention (Table 1).

Table 1. Baseline values of measured variables

\begin{tabular}{|c|c|c|c|c|}
\hline Variables & Conditions & Mean \pm SD & $\begin{array}{c}F \\
(\mathrm{df}(3.92))\end{array}$ & $\begin{array}{l}\text { Signifi- } \\
\text { cance }\end{array}$ \\
\hline \multirow{4}{*}{$\begin{array}{l}\text { Hooper } \\
\text { index (AU) }\end{array}$} & AA & $9.4 \pm 4.3$ & \multirow{4}{*}{0.32} & \multirow{4}{*}{0.81} \\
\hline & $\mathrm{AC}$ & $9.5 \pm 2.9$ & & \\
\hline & $\mathrm{CA}$ & $10.7 \pm 3.7$ & & \\
\hline & $\mathrm{CC}$ & $10.5 \pm 4.8$ & & \\
\hline \multirow{4}{*}{$\begin{array}{l}\text { First day } \\
\operatorname{lnRMSSD} \\
(\log )\end{array}$} & AA & $4.1 \pm 0.5$ & \multirow{4}{*}{0.55} & \multirow{4}{*}{0.65} \\
\hline & $\mathrm{AC}$ & $4.1 \pm 0.5$ & & \\
\hline & $\mathrm{CA}$ & $4.0 \pm 0.4$ & & \\
\hline & $\mathrm{CC}$ & $3.9 \pm 0.4$ & & \\
\hline \multirow{4}{*}{$\begin{array}{l}\text { First day } \\
\text { SDNN (ms) }\end{array}$} & AA & $109.6 \pm 0.4$ & \multirow{4}{*}{1.09} & \multirow{4}{*}{0.37} \\
\hline & $\mathrm{AC}$ & $108.01 \pm 55.2$ & & \\
\hline & $\mathrm{CA}$ & $86.8 \pm 39.1$ & & \\
\hline & $\mathrm{CC}$ & $84.6 \pm 38.6$ & & \\
\hline \multirow{4}{*}{$\begin{array}{l}\text { First day } \\
\text { RPE (AU) }\end{array}$} & $\mathrm{AA}$ & $3.9 \pm 1.4$ & \multirow{4}{*}{0.39} & \multirow{4}{*}{0.76} \\
\hline & $\mathrm{AC}$ & $4.1 \pm 1.6$ & & \\
\hline & $\mathrm{CA}$ & $4.5 \pm 1.3$ & & \\
\hline & $\mathrm{CC}$ & $4.0 \pm 1.7$ & & \\
\hline \multirow{4}{*}{$\begin{array}{l}\text { Pre-SSGs } \\
\operatorname{lnRMSSD} \\
(\log )\end{array}$} & AA & $2.5 \pm 0.8$ & \multirow{4}{*}{1.45} & \multirow{4}{*}{0.24} \\
\hline & $\mathrm{AC}$ & $2.4 \pm 0.8$ & & \\
\hline & $\mathrm{CA}$ & $2.6 \pm 0.6$ & & \\
\hline & $\mathrm{CC}$ & $3.0 \pm 0.7$ & & \\
\hline \multirow{4}{*}{$\begin{array}{l}\text { Pre-SSGs } \\
\text { SDNN (ms) }\end{array}$} & AA & $28.4 \pm 12.7$ & \multirow{4}{*}{0.79} & \multirow{4}{*}{0.51} \\
\hline & $\mathrm{AC}$ & $30.1 \pm 12.3$ & & \\
\hline & CA & $33.5 \pm 17.7$ & & \\
\hline & $\mathrm{CC}$ & $39.4 \pm 28.3$ & & \\
\hline \multirow{4}{*}{$\begin{array}{l}\text { Pre-recovery } \\
\text { speed (s) }\end{array}$} & AA & $2.21 \pm 0.1$ & \multirow{4}{*}{0.81} & \multirow{4}{*}{0.50} \\
\hline & $\mathrm{AC}$ & $2.1 \pm 0.1$ & & \\
\hline & $\mathrm{CA}$ & $2.1 \pm 0.1$ & & \\
\hline & $\mathrm{CC}$ & $2.2 \pm 0.1$ & & \\
\hline
\end{tabular}

AA - active-active strategy, AC - active-CWI strategy, CA - CWI-active strategy, CC - CWI-CWI strategy, CWI - cold-water immersion, AU - astronomical unit, lnRMSSD - log-transformed root mean square of successive R-R intervals, SDNN - standard deviation of normal R-R intervals, RPE - rate of perceived exertion, SSG - small-sided game

\section{Participants}

A total of 24 semi-professional club and collegiate male soccer players from a first division Iranian National League took part in the present study (Table 2). The players' training routine included 3 training sessions (90 minutes per session) and 1 match per week. The inclusion criteria were: (1) at least 5 years of training experience; (2) no recent (within 4 weeks prior to the study) muscular or skeletomuscular injuries; (3) non-smoking status; (4) no use of sports supplements, anti-inflammatory drugs, or medication within at least 6 months prior to the study; (5) not travelling for at least a week prior to the study; and (6) sleeping at least 7-8 hours per day. Exclusion criteria involved cardiovascular diseases, sleep disorders, and other risk factors based on a doctor's examination. All participants were familiar with SSGs and had frequently participated in such training. Furthermore, all players were fully acquainted with the experimental procedures. The research followed the ethical standards of the Declaration of Helsinki for studies in humans [16]. The study lasted for 4 weeks. Each week consisted of 2 data collection sessions (Monday and Wednesday). The participants were randomly assigned to 4 experimental conditions $(n=6$ in each condition). They all completed the 4 recovery strategies during the 4 -week study.

Table 2. Anthropometric characteristics of participants

\begin{tabular}{lc}
\hline Characteristics & Mean \pm SD \\
\hline Age (years) & $22.3 \pm 2.6$ \\
Weight $(\mathrm{kg})$ & $75.3 \pm 4.2$ \\
Height $(\mathrm{cm})$ & $176.0 \pm 12.0$ \\
Body mass index $\left(\mathrm{kg} / \mathrm{m}^{2}\right)$ & $24.3 \pm 1.1$ \\
\hline
\end{tabular}

\section{Small-sided games}

We used 3 vs. 3 ball possession SSGs played on an $18 \times 20 \mathrm{~m}$ pitch (area per player: $60 \mathrm{~m}^{2}$ ) and a training regimen of $4 \times 4$ minutes interspaced by $3 \mathrm{~min}$ utes of recovery [15]. The SSGs were performed after a standardized warm-up protocol of 4 minutes consisting of light running, mobility, and neuromuscular movements. The SSGs were carried out with the command to preserve collective ownership of the ball as long as possible [15]. To reduce disturbances when the ball left the pitch, spare balls were kept around the pitch, with 4 supporting players situated outside the playing area to put a spare ball into play when needed. Additionally, coaches constantly provided ver- 
bal encouragement to the players throughout the SSGs. The players were permitted to drink water during all recovery times during the SSGs [15].

\section{Recovery strategies and procedures}

On the first morning, before the training session (immediately after waking up), the participants assumed a sitting position for 1 minute and then measured HRV for 1 minute using a Polar H7 pulse rate monitor. Also, to reflect the player's physical state, the numbers corresponding to the Hooper questionnaire items (sleep, fatigue, pain, and stress) were reported and sent to the researcher with the WhatsApp software. The HRV index was measured for 1 minute; after the SSGs, the players' HRV scores were assessed again while seated by using a Polar $\mathrm{H} 7$ pulse rate monitor and the Elite HRV software (Figure 1).

To reflect the players' condition, the numbers corresponding to the Hooper questionnaire items (sleep, fatigue, pain, and stress) were reported and sent to the researcher through the WhatsApp software. The participants followed the AR strategy with $40-50 \%$ of HRmax for 10 minutes. After the subsequent rest period, they measured their HRV index scores using Polar H7 pulse rate monitors and the Elite HRV software; HRV index scores were measured in 1 minute while the players were seated. Also, training intensity was evaluated with the RPE index (CR10 Borg's scale) [17]. On the second day (immediately after waking up), the players measured their HRV index using a Polar H7 pulse rate monitor and the Elite HRV software for 1 minute while seated (Figure 1).
Moreover, to reflect the participants' conditions, the numbers corresponding to the Hooper questionnaire items (sleep, fatigue, pain, and stress) were recorded and sent to the researcher through the WhatsApp software. Then, in the afternoon (before the start of the training session), the players measured their HRV while seated using a Polar H7 pulse rate monitor and the Elite HRV software. Also, to reflect their physical state, the numbers corresponding to the Hooper index items (sleep, fatigue, pain, and stress) were recorded and sent to the researcher through the WhatsApp software.

On this day (24 hours after the training), the players used the CWI recovery strategy $\left(10-12^{\circ} \mathrm{C}\right.$ for 10 minutes, immersion to the iliac spine level). Then, after recovery, they measured their HRV index again for 1 minute using a Polar $\mathrm{H} 7$ pulse rate monitor and the Elite HRV software. During the morning session on the third day (immediately after waking up), the participants measured their HRV index using a Polar H7 pulse rate monitor and the Elite HRV software for 1 minute. Also, to reflect the players' condition, the numbers corresponding to the Hooper index (sleep, fatigue, pain, and stress) were recorded and sent to the researcher through the WhatsApp software. Then, in the afternoon of the same day (before the training), the players measured their HRV using a Polar H7 pulse rate monitor and the Elite HRV software for 1 minute (Figure 1).

In addition, to reflect their physical state, the numbers corresponding to the Hooper index items (sleep, fatigue, pain, and stress) were recorded and sent to the researcher through the WhatsApp software. On

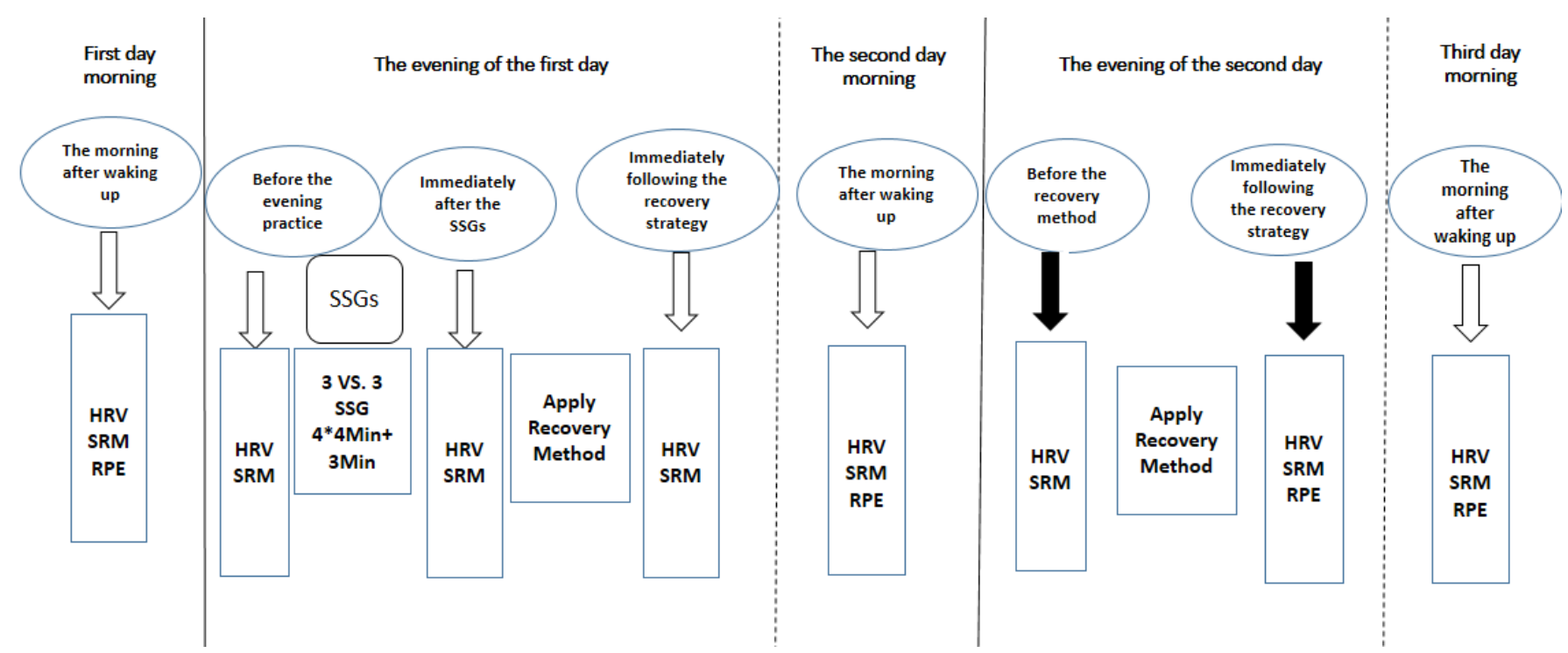

HRV - heart rate variability, SRM - self-reported measurement, RPE - rate of perceived exertion, SSG - small-sided game

Figure 1. Experimental protocol 
M.K. Baseri, J. Reisi, R. Bagheri, F.M. Clemente, Active and cold-water immersion recovery strategies

this day, the players performed a $20-\mathrm{m}$ sprinting test. During the submaximal test, they tightened their pacemakers and recorded changes in HR, sprint distance, calorie intake, and other data using the Polar Beat software. The participants performed 3 repetitions of the $20-\mathrm{m}$ sprinting test, with a 2-minute rest period between the sets. The time recorder automatically verified the players' test times.

\section{Self-reported measurement}

Subjective wellness indices were gathered by utilizing an altered psychological questionnaire in accordance with the commendations by Hooper and Mackinnon [18, 19]. The questionnaire comprises subsets of perceived quality of sleep, stress, muscular fatigue, or soreness, with each item counted on a 7-point scale (with 1 and 7 representing 'very, very good' and 'very, very poor' wellness ratings, respectively). Overall wellness (Hooper index) was established through the summation of the 4 scores [18]. The players were previously familiarized with the questionnaire and the scale. During the data collection, the scores were provided individually to prevent participants from hearing one another's responses, thus maximizing the accuracy of the scores.

\section{0-m sprint test}

The participants performed a warm-up protocol lasting 4 minutes and consisting of light running, mobility, and neuromuscular movements. They stood $1 \mathrm{~m}$ apart before the first speed gate and ran at a maximum speed with a steering wheel. Upon skipping the first gate speedometer, the small speedometer monitor (Arena Gear, RM-501-TrackNField-2G; USA) automatically started, and after the last gate was passed at the end of the 20-m course, the monitor automatically stopped. The speed was recorded in milliseconds. There was a 2-minute break between the trial attempts, and all 3 times were recorded. The best score was assumed and used for the statistical analysis. The test was performed on the first day before the SSGs and on the third day in the afternoon (around 4-6 pm) at a temperature of $24^{\circ} \mathrm{C}$.

\section{Heart rate variability}

For measuring HRV, each participant used an Elite HRV smartphone application and a Polar H7 HR monitor (Polar Electro Oy; Kempele, Finland) with a sampling frequency of $1000 \mathrm{~Hz}$. Raw data of R-R intervals were handled through the Elite HRV to calculate HRV measures [18]. These resources have lately been authenticated in a study showing a high conformity with the Kubios HRV 2.2 software for calculating the root mean square of successive differences (RMSSD) [19]. Recording measures comprised of a supine HRV evaluation conducted by each participant with their smartphone following individual waking times (i.e., the same individual time). HRV records continued for 1 minute and were headed by a 1.5 -minute steadiness period [18]. All HRV records were fulfilled under impulsive breathing situations to avoid inconsistencies in the outcomes for the log-transformed root mean square of successive R-R intervals (lnRMSSD) [20]. The standard deviation of normal R-R intervals (SDNN) and lnRMSSD were collected with the Elite HRV software [18]. The measurement was taken in the morning in the house and in the afternoon in the field.

\section{Rate of perceived exertion}

The players' RPE values (CR10 Borg's scale) [5] were assessed before the study and 24 and 48 hours after the recovery strategies. The participants were required to report the RPE of the game. In addition, they had been habituated with the RPE procedure in the earlier week in a theoretical session. The scores were provided individually.

\section{Statistical analysis}

Data normality and homogeneity were preliminarily tested by using the Kolmogorov-Smirnov test and Levene's test, respectively. After verifying the assumptions of normality and homogeneity ( $p>0.05)$, a mixed model of ANOVA was applied to test the within- and between-experimental conditions differences. Statistical significance was set at $p<0.05$. The Bonferroni post-hoc test was utilized for pairwise comparisons between experimental conditions. The magnitude of the changes was determined by executing partial eta squared $\left(\eta^{2}\right)$ in ANOVA and the standardized effect size of Cohen in pairwise comparisons. Ferguson's classification for partial eta squared $\left(\eta^{2}\right)$ was utilized as follows: no effect $\left(\eta^{2}<0.04\right)$, minimum effect $\left(0.04 \leq \eta^{2}\right.$ $<0.25)$, moderate effect $\left(0.25 \leq \eta^{2}<0.64\right)$, and strong effect $\left(\eta^{2} \geq 0.64\right)$ [5]. All statistical analyses were performed with the SPSS software (version 24; IBM, Chicago, USA).

\section{Ethical approval}

The research related to human use has complied with all the relevant national regulations and institu- 
tional policies, has followed the tenets of the Declaration of Helsinki, and has been approved by the $\mathrm{Hu}$ man Subject Committee of the University of Isfahan (IR.UI.REC.1397.135).

\section{Informed consent}

Informed consent has been obtained from all individuals included in this study.
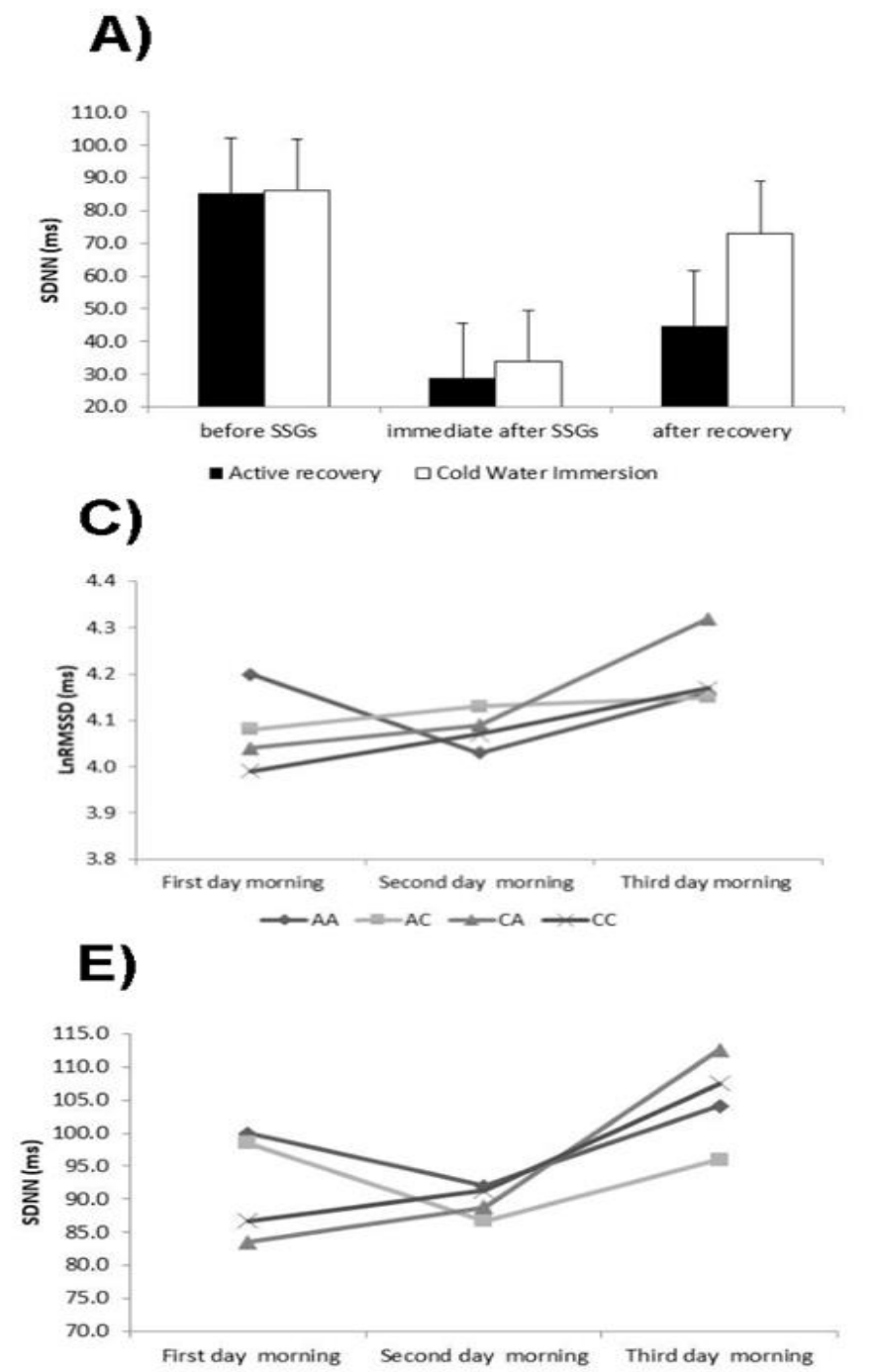

\section{G)}

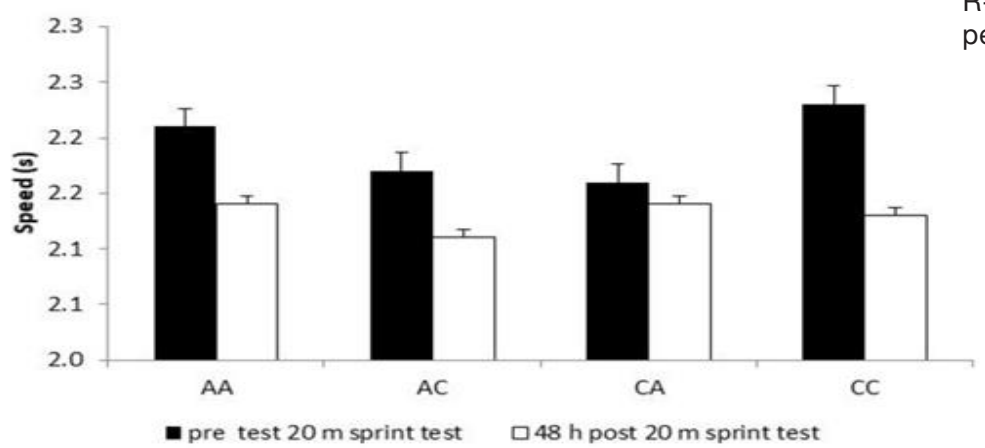

\section{Results}

Figure 2A represents the SDNN index (standard deviation of all NN intervals for all 1-minute segments of the total recording) changes between the AR and CWI recovery strategies. The between-experimental conditions analysis revealed a significant difference in SDNN HR variations between AR and CWI $(F=4.86$, $p=0.03, \eta^{2}=0.31$, moderate effect). Thus, CWI showed
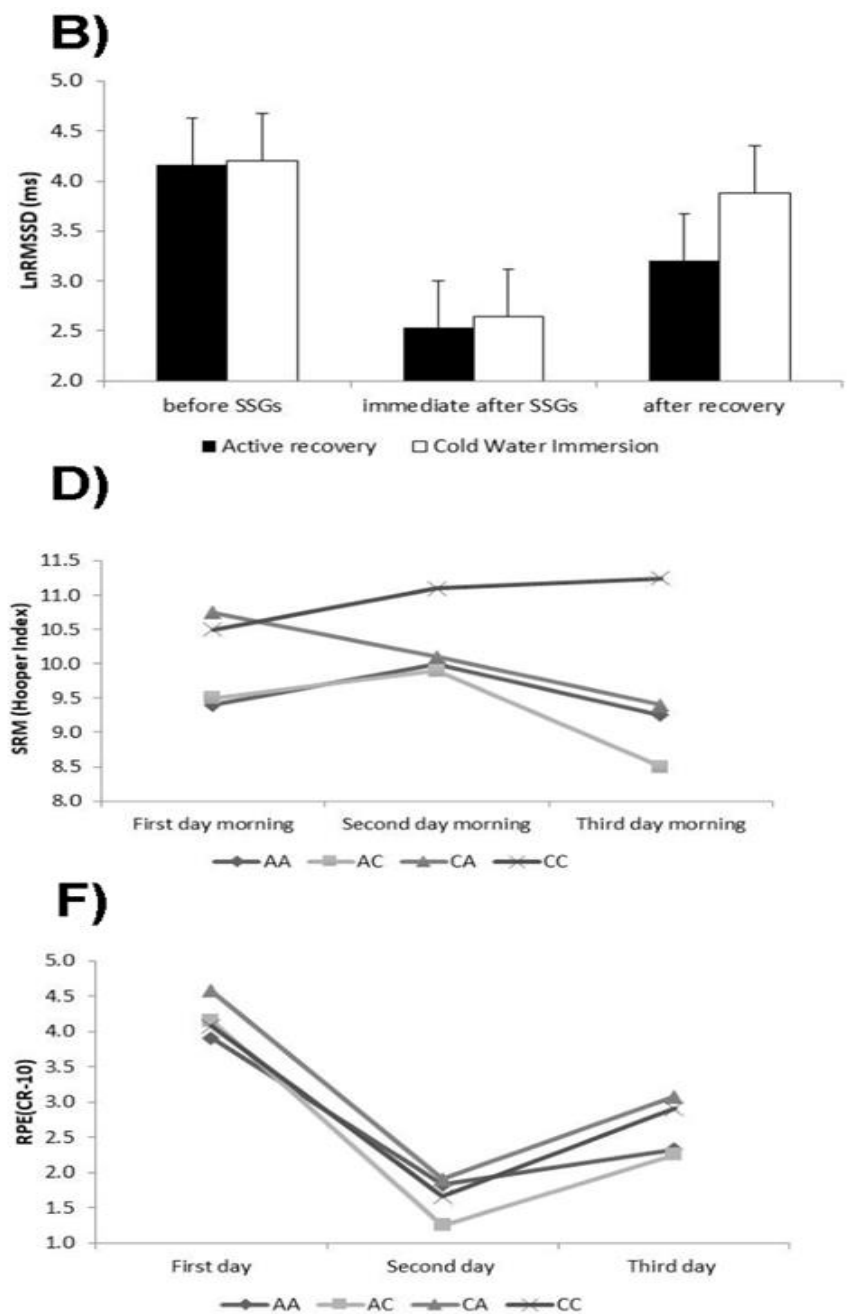

$A A$ - active-active strategy, $A C$ - active-CWI strategy, CA - CWI-active strategy, CC - CWI-CWI strategy, CWI - cold-water immersion, SDNN - standard deviation of normal R-R intervals, SSG - small-sided game, InRMSSD - log-transformed root mean square of successive R-R intervals, SRM - self-reported measurement, RPE - rate of perceived exertion

Figure 2. (A) SDNN index changes between active and CWI recovery, (B) lnRMSSD index changes between active and CWI recovery, $(\mathrm{C}) \mathrm{lnRMSSD}$ index changes between different strategies, (D) Hopper index changes in the morning between different strategies, $(\mathrm{E})$ variations of SDNN index between different strategies, (F) changes in the perceived pressure index of exercise between different strategies, $(\mathrm{G})$ changes in $20-\mathrm{m}$ sprint test between different strategies 
M.K. Baseri, J. Reisi, R. Bagheri, F.M. Clemente, Active and cold-water immersion recovery strategies

a better effect (mean difference: 23.2; 242.2\%) than $\mathrm{AR}$ in restoring the impaired vagal-related $\mathrm{HRV}$ indices observed after SSGs. As for the within-experimental condition changes $\left(F=60.82, p=0.001, \eta^{2}=0.85\right.$, strong effect), significant differences were detected between the status before SSGs and immediately after SSGs ( $p=0.001$ ), as well as between the status before SSGs and immediately after recovery $(p=0.001)$.

There was a significant difference in lnRMSSD HR variations (Figure $2 \mathrm{~B}$ ) between the $\mathrm{AR}$ and $\mathrm{CWI}$ recovery strategies $\left(F=2.41, p=0.033, \eta^{2}=0.29\right.$, moderate effect). Thus, CWI showed a better effect (mean difference: $0.81 ; 185.7 \%$ ) than AR in restoring the impaired vagal-related HRV indices observed after SSGs. As for the within-experimental condition changes $\left(F=127.9, p=0.001, \eta^{2}=0.74\right.$, strong effect $)$, significant differences were noted between the status before SSGs and immediately after SSGs ( $p=0.001)$, as well as between the status before SSGs and immediately after recovery $(p=0.001)$.

In addition, there was no significant difference in lnRMSSD HR variations (Figure 2C) between the different strategies $\left(F=2.54, p=0.065, \eta^{2}=0.04\right)$. As for the within-experimental condition changes $(F=$ $1.96, p=0.14, \eta^{2}=0.04$, no effect), no significant difference was determined between the first and the second day $(p=0.47)$ or between the second and the third day after recovery $(p=0.17)$.

Furthermore, there was no significant difference in Hopper index changes (Figure 2D) between the different strategies $\left(F=0.291, p=0.832, \eta^{2}=0.11\right.$, minimum effect). As for the within-experimental condition changes $\left(F=0.81, p=0.22, \eta^{2}=0.02\right.$, no effect), significant differences were not noted between the first and the second day $\left(p=0.66, \eta^{2}=0.03\right)$ or between the second and the third day after recovery ( $p=0.81$, $\eta^{2}=0.02$ ).

There was no significant difference in SDNN HR changes (Figure 2E) between the different strategies $\left(F=2.73, p=0.055, \eta^{2}=0.07\right.$, no effect). However, the CA strategy seems more effective than other strategies in reducing HR. In the within-experimental condition analysis $\left(F=2.55, p=0.08, \eta^{2}=0.05\right.$, moderate effect), no significant differences were observed between the first and the second day $\left(p=0.72, \eta^{2}=0.02\right)$ or between the second and the third day, and immediately after recovery $\left(p=0.052, \eta^{2}=0.03\right)$.

Additionally, no significant difference was noted in changes of the perception pressure index (Figure $2 \mathrm{~F}$ ) between the different strategies $(F=978, p=0.41$, $\eta^{2}=0.06$, minimum effect). In the within-experimental conditions analysis $\left(F=50.05, p=0.001, \eta^{2}=0.53\right.$, moderate effect), significant differences were noted between the first and the second day $\left(p=0.001, \eta^{2}=\right.$ 0.58 moderate effect), between the second and the third day, and immediately after recovery $\left(p=0.001, \eta^{2}=\right.$ 0.44 , moderate effect).

Moreover, there was no significant difference between the changes in the 20-m sprint test (Figure 2G) between the different strategies $(F=0361, p=0.78$, $\eta^{2}=0.02$, no effect). In the final within-experimental conditions analysis $\left(F=18.8, p=0.001, \eta^{2}=0.29\right.$, moderate effect), significant differences were noted between the status before and. 48 hours after the $20-\mathrm{m}$ sprint test ( $p=0.001, \eta^{2}=0.37$, moderate effect).

\section{Discussion}

The purpose of this investigation was to compare the effects of 4 recovery strategies in semi-professional soccer players. The results showed a significant difference between AR and CWI in SDNN and InRMSSD HR variations. Thus, CWI showed a greater effect than $\mathrm{AR}$ in restoring the decreased vagal-related HRV indices observed immediately after SSGs. However, the results revealed no significant difference between the lnRMSSD and SDNN HRV indices, Hooper index, RPE, or the 20-m sprint test in the applied strategies. Therefore, the main findings of this study were that (1) HRV indices were significantly increased after CWI and (2) none of the strategies caused significant changes in the measured variables.

We used HRV indices to noninvasively assess cardiac autonomic function because the straight evaluation of cardiac parasympathetic nerve activity (soon after the training) was not achievable. The limitations of using HRV indices are well known [21, 22]. A decrease in cardiac vagal activity after training can cause ischemic heart disease, which is the pathogenesis of malignant ventricular arrhythmias and sudden cardiac mortality [21]. Buchheit et al. [21] investigated the immediate effect of using a CWI strategy on postexercise parasympathetic reactivation and showed that CWI meaningfully restored the lessened vagal-related HRV indices after super-maximal training. Thus, CWI might be an applicable tool that can quicken parasympathetic reactivation during the period immediately after super-maximal training [21].

Al Haddad et al. [23] investigated the effects of cold and thermoneutral water immersion on post-training parasympathetic reactivation inferred from HR recovery and HRV measures [23]. In agreement with our findings, they revealed that water immersion was an efficient strategy for approximately generating post- 
training parasympathetic activity, with cold immersion temperatures liable to be more applicable to increase parasympathetic activity [23]. In addition, Bastos et al. [10] examined the effects of CWI and AR on postexercise HRV [10], indicating improvements in postexercise cardiac autonomic function when compared with $\mathrm{AR}$ and passive recovery.

Indeed, SSGs triggered a significant reduction in vagal-related HRV indices quickly after the termination of exercise. However, we could not establish the precise mechanisms by which the CWI strategy influenced the autonomic system after training; this matter necessitates future examination. However, our frequently utilized recovery $\left(10\right.$ minutes at $10-12^{\circ} \mathrm{C}$, immersion to the iliac spine level) caused parasympathetic supremacy that was mirrored by the noted bradycardia and enhancement of HRV indices.

Our results approve that CWI increases cardiac parasympathetic control [21]. Consequently, the efficiency of CWI $\left(10\right.$ minutes at $10-12^{\circ} \mathrm{C}$, immersion to the iliac spine level) in restoring parasympathetic modulation depends on the advantageous connections among the 2 branches of the autonomic nervous system that are concurrently responsible for thermoregulation, cardiovascular stability, and homeostasis restoration. The current results confirm that CWI can be utilized to fleetingly improve the cardioprotective background at the minimum after SSGs [21].

Generally, the temperature of the water and hydrostatic pressure have been identified owing to the applicable agents on the regenerative advantages of this strategy [24]. The conceivable changes produced through this method include modifications in the intracellular and intravascular fluid, diminished muscular oedema, augmented cardiac adaptation [24], improved blood flow, and the removal of hydrogen ions, lactate, etc. [24]. Furthermore, psychological benefits have been illustrated (e.g., the analgesic effect due to changes in the perception of pain and discomfort [24] or fatigue reduction throughout the immersion). CWI decreases cardiac response and increases cardiac peripheral resistance, oxygen consumption, and metabolism as the body works to maintain its core temperature [24].

Alternatively, AR can be performed closely after or during a subsequent training session. Although it is a commonly employed recovery strategy among soccer players with divergent training experience, there is no strong evidence to confirm its beneficial effects [25]. Numerous outcomes have been outlined, in both the short term and long term, on the recovery process in soccer (intersession) [24]. In the short term, low-inten- sity endurance exercise appears to produce a quicker excretion of lactate [24], which is used by the liver, as well as by cardiac and skeletal muscles to produce ATP through the gluconeogenesis cycle [24]. Additional likely favourable results are fewer unexpected drops in core temperature that decrease the arousal level of the central nervous system, thereby leading to higher sleep quality after a match [24]. Conversely, various negative influences of the AR strategy have been explained, such as glycogen depletion within skeletal muscles [24]. Although low-intensity endurance exercise seems to have encouraging effects on lactate excretion, its impact on sports performance is not conclusive. Consequently, whether this recovery strategy reduces acute fatigue after a match or training session is arguable and continues to be an engaging topic of research [24].

Moreover, there was no significant difference between the changes in the 20-m sprint test performance among the different strategies. Regarding the withinexperimental condition changes, significant differences were noted between pre-test data and data collected 48 hours after the test. It seems that the improvement in the 20-m sprint test performance is due to the optimal effectiveness of each recovery strategy.

To date, few studies have explored the use of recovery strategies and their combinations [7, 26-28]. Former investigations confirm the benefits of various recovery strategies for function-related factors [26]. CWI seems to decrease muscle damage and stiffness after high-intensity eccentric exercise. Meanwhile, endurance exercise and electrostimulation appear to reduce muscle pain [26]. In addition, contrast baths and low-intensity endurance exercise seem to diminish the muscle damage marker (e.g., creatine kinase) concentrations. Nevertheless, on the basis of the outcomes presented here, we cannot conclude that there is only a single efficient recovery strategy. Kinugasa and Kilding [27] also showed that a combination of recovery techniques, e.g., cryotherapy and AR, significantly improved the rate of recovery in soccer players [27].

According to the results of this study, there was no significant decrease in the Hooper index responses in any of the strategies. In other words, all 4 strategies led to equal improvements in Hooper index responses. The decrease in Hooper index scores can be considered a function of fatigue decrements, as the self-reported measurement (Hooper index) is a combination of 4 factors that measure sleep, muscle pain, stress, and fatigue. The present study is novel in terms of exploring the impact of the recovery strategies on Hooper index responses. 
M.K. Baseri, J. Reisi, R. Bagheri, F.M. Clemente, Active and cold-water immersion recovery strategies

In addition, in our study, no significant difference was noted between all strategies regarding RPE. We used the CR10 Borg's scale to measure muscle soreness with 4 different recovery strategies [26, 29]. Although this scale is usually utilized to measure localized muscle pain [26], 1 study found advantageous effects of its application to evaluate recovery after training or after recovery strategies. Elias et al. [9] investigated the efficiency of a single exposure to 14 minutes of CWI and CWT in post-training recovery among Australian soccer players [9] and revealed attenuated muscle soreness after both CWT and CWI. CWT reduced soreness at 1 and 48 hours, while CWI reduced soreness close to baseline levels at 1 hour and reduced muscle soreness after 48 hours. Both strategies were effective in moderating perceived fatigue. Fatigue was attenuated by CWT at 1 and 48 hours. CWI also reduced fatigue after 1 and 24 hours, while reducing fatigue to pre-training levels after 48 hours [9].

In our study, CR10 Borg's scale scores were elevated in all experimental conditions, showing that the global perceived muscle soreness in the lower extremity musculature increased at the end of the training session. Capodaglio [29] used the CR10 pain scale to assess - in 4-minute intervals - perceived muscle soreness of the upper body during and after a highintensity arm ergometer test [26, 29]. It is likely that the utilization of continuous running for 8 minutes during recovery destructively influences athletes' perceptions of muscle pain in comparison with strategies that employ relaxation stretch techniques without requiring any motor activity. For example, the stretching and leg raise technique involves supine resting with the legs elevated, which promotes venous return and reduces pain in the lower limbs [26].

The main objective of this evaluation was to compare the effects of 4 recovery strategies among semiprofessional soccer players in terms of HR-related indices. However, our training procedure did not allow us to decipher the respective influences of immersion vs. cold exposure on post-exercise parasympathetic reactivation. Nevertheless, the present study has the advantage of being performed in 4 microcycles; as such, the data reflect actual specific loads. This, paired with the capability to screen the variables in an organized and standardized way, further strengthens the conclusions presented in this study. The sample size of the research represents a potential limitation with regard to the generalizability of the findings.

\section{Conclusions}

It would be beneficial for soccer players to use CWI (10 minutes at $10-12^{\circ} \mathrm{C}$, immersion to the iliac spine level) to relieve the metabolic stress, especially in the cardiac muscle, immediately after high-intensity workouts such as SSGs. It seems that the order of the recovery strategies has no effect on the speed of recovery after SSGs.

\section{Disclosure statement}

No author has any financial interest or received any financial benefit from this research.

\section{Conflict of interest}

The authors state no conflict of interest.

\section{References}

1. Nédélec M, McCall A, Carling C, Legall F, Berthoin S, Dupont G. Recovery in soccer: part I - post-match fatigue and time course of recovery. Sports Med. 2012; 42(12):997-1015; doi: 10.2165/11635270-00000000 0-00000.

2. Romagnoli M, Sanchis-Gomar F, Alis R, Risso-Ballester J, Bosio A, Graziani RL, et al. Changes in muscle damage, inflammation, and fatigue-related parameters in young elite soccer players after a match. J Sports Med Phys Fitness. 2016;56(10):1198-1205.

3. Thomas K, Dent J, Howatson G, Goodall S. Etiology and recovery of neuromuscular fatigue after simulated soccer match play. Med Sci Sports Exerc. 2017;49(5): 955-964; doi: 10.1249/MSS.0000000000001196.

4. Jeong T-S, Reilly T, Morton J, Bae S-W, Drust B. Quantification of the physiological loading of one week of "pre-season" and one week of "in-season" training in professional soccer players. J Sports Sci. 2011;29(11): 1161-1166; doi: 10.1080/02640414.2011.583671.

5. Clemente FM, Nikolaidis PT, van der Linden CMI, Silva B. Effects of small-sided soccer games on internal and external load and lower limb power: a pilot study in collegiate players. Hum Mov. 2017;18(1):50-57; doi: 10.1515/humo-2017-0007.

6. Clemente FM, Sarmento H. The effects of small-sided soccer games on technical actions and skills: a systematic review. Hum Mov. 2020;21(3):100-119; doi: 10.5114/hm.2020.93014.

7. Barnett A. Using recovery modalities between training sessions in elite athletes: does it help? Sports Med. 2006;36(9):781-796; doi: 10.2165/00007256-200636 090-00005.

8. Andersson H, Raastad T, Nilsson J, Paulsen G, Garthe I, Kadi F. Neuromuscular fatigue and recovery in elite female soccer: effects of active recovery. Med Sci Sports Exerc. 2008;40(2):372-380; doi: 10.1249/mss.0b013e $31815 b 8497$. 
9. Elias GP, Varley MC, Wyckelsma VL, McKenna MJ, Minahan CL, Aughey RJ. Effects of water immersion on posttraining recovery in Australian footballers. Int J Sports Physiol Perform. 2012;7(4):357-366; doi: 10.1123/ijspp.7.4.357.

10. Bastos FN, Vanderlei LCM, Nakamura FY, Bertollo M, Godoy MF, Hoshi RA, et al. Effects of cold water immersion and active recovery on post-exercise heart rate variability. Int J Sports Med. 2012;33(11):873879; doi: 10.1055/s-0032-1301905.

11. Pooley S, Spendiff O, Allen M, Moir HJ. Comparative efficacy of active recovery and cold water immersion as post-match recovery interventions in elite youth soccer. J Sports Sci. 2020;38(11-12):1423-1431; doi: 10.1080/02640414.2019.1660448.

12. Mascarin RB, De Andrade VL, Barbieri RA, Loures JP, Kalva-Filho CA, Papoti M. Dynamics of recovery of physiological parameters after a small-sided game in women soccer players. Front Physiol. 2018;9:887; doi: 10.3389/fphys.2018.00887.

13. Clemente FM, Lourenço Martins FM, Mendes RS. Developing aerobic and anaerobic fitness using smallsided soccer games: methodological proposals. Strength Cond J. 2014;36(3):76-87; doi: 10.1519/SSC.000000 0000000063.

14. Sparkes W, Turner A, Weston M, Russell M, Johnston M, Kilduff L. Neuromuscular, biochemical, endocrine, and mood responses to small-sided games' training in professional soccer. J Strength Cond Res. 2018;32(9): 2569-2576; doi: 10.1519/JSC.0000000000002424.

15. Köklü Y, Alemdaroğlu U, Dellal A, Wong DP. Effect of different recovery durations between bouts in 3-a-side games on youth soccer players' physiological responses and technical activities. J Sports Med Phys Fitness. 2015;55(5):430-438.

16. World Medical Association. World Medical Association Declaration of Helsinki: ethical principles for medical research involving human subjects. JAMA. 2013; 310(20):2191-2194; doi: 10.1001/jama.2013.281053.

17. Borg G. Borg's perceived exertion and pain scales. Champaign: Human Kinetics; 1998.

18. Flatt AA, Esco MR, Nakamura FY. Individual heart rate variability responses to preseason training in high level female soccer players. J Strength Cond Res. 2017; 31(2):531-538; doi: 10.1519/JSC.0000000000001482.

19. Rabbani A, Baseri MK, Reisi J, Clemente FM, Kargarfard M. Monitoring collegiate soccer players during a congested match schedule: heart rate variability versus subjective wellness measures. Physiol Behav. 2018; 194:527-531; doi: 10.1016/j.physbeh.2018.07.001.

20. Wong A, Bergen D, Nordvall M, Allnutt A, Bagheri R. Cardiac autonomic and blood pressure responses to an acute session of battling ropes exercise. Physiol Behav. 2020;227:113167; doi: 10.1016/j.physbeh.2020.113167.

21. Buchheit M, Peiffer JJ, Abbiss CR, Laursen PB. Effect of cold water immersion on postexercise parasympathetic reactivation. Am J Physiol Heart Circ Physiol.
2009;296(2):H421-H427; doi: 10.1152/ajpheart.01017. 2008.

22. Wong A, Figueroa A, Fischer SM, Bagheri R, Park S-Y. The effects of mat Pilates training on vascular function and body fatness in obese young women with elevated blood pressure. Am J Hypertens. 2020;33(6):563-569; doi: 10.1093/ajh/hpaa026.

23. Al Haddad H, Laursen PB, Chollet D, Lemaitre F, Ahmaidi S, Buchheit M. Effect of cold or thermoneutral water immersion on post-exercise heart rate recovery and heart rate variability indices. Auton Neurosci. 2010; 156(1-2):111-116; doi: 10.1016/j.autneu.2010.03.017.

24. Rey E, Padrón-Cabo A, Barcala-Furelos R, Casamichana D, Romo-Pérez V. Practical active and passive recovery strategies for soccer players. Strength Cond J. 2018;40(3):45-57; doi: 10.1519/SSC.00000000000 00247.

25. Rey E, Lago-Peñas C, Lago-Ballesteros J, Casáis L. The effect of recovery strategies on contractile properties using tensiomyography and perceived muscle soreness in professional soccer players. J Strength Cond Res. 2012;26(11):3081-3088; doi: 10.1519/JSC.0b013 e3182470d33.

26. García-Concepción MA, Peinado AB, Paredes Hernández V, Alvero-Cruz JR. Efficacy of different recovery strategies in elite football players. Rev Int Med Cienc Act Fis Deporte. 2015;15(58):355-369; doi: 10.15366/ RIMCAFD2015.58.010.

27. Kinugasa T, Kilding AE. A comparison of post-match recovery strategies in youth soccer players. J Strength Cond Res. 2009;23(5):1402-1407; doi: 10.1519/JSC. 0b013e3181a0226a.

28. Monedero J, Donne B. Effect of recovery interventions on lactate removal and subsequent performance. Int $\mathrm{J}$ Sports Med. 2000;21(8):593-597; doi: 10.1055/s-20008488.

29. Capodaglio EM. Comparison between the CR10 Borg's scale and the VAS (visual analogue scale) during an arm-cranking exercise. J Occup Rehabil. 2001;11(2): 69-74; doi: 10.1023/a:1016649717326. 